\title{
Fabrication of miniature elastomer lenses with programmable liquid mold for smartphone microscopy: curing polydimethylsiloxane with in situ curvature control
}

Bhuvaneshwari Karunakaran

Joseph Tharion

Arvind Ramrao Dhawangale

Debjani Paul

Soumyo Mukherji 


\title{
Fabrication of miniature elastomer lenses with programmable liquid mold for smartphone microscopy: curing polydimethylsiloxane with in situ curvature control
}

\author{
Bhuvaneshwari Karunakaran, ${ }^{\mathrm{a}}$ Joseph Tharion, ${ }^{\mathrm{b}}$ Arvind Ramrao Dhawangale, ${ }^{\mathrm{a}}$ Debjani Paul, ${ }^{\mathrm{a}}$ and \\ Soumyo Mukherjia, \\ andian Institute of Technology Bombay, Department of Biosciences and Bioengineering, Powai, Mumbai, India \\ 'Indian Institute of Technology Bombay, Department of Chemistry, Powai, Mumbai, India
}

\begin{abstract}
Miniature lenses can transform commercial imaging systems, e.g., smartphones and webcams, into powerful, low-cost, handheld microscopes. To date, the reproducible fabrication of polymer lenses is still a challenge as they require controlled dispensing of viscous liquid. This paper reports a reproducible lens fabrication technique using liquid mold with programmable curvature and off-the-shelf materials. The lens curvature is controlled during fabrication by tuning the curvature of an interface of two immiscible liquids [polydimethylsiloxane (PDMS) and glycerol]. The curvature control is implemented using a visual feedback system, which includes a software-based guiding system to produce lenses of desired curvature. The technique allows PDMS lens fabrication of a wide range of sizes and focal lengths, within $20 \mathrm{~min}$. The fabrication of two lens diameters: 1 and $5 \mathrm{~mm}$ with focal lengths ranging between 1.2 and $11 \mathrm{~mm}$ are demonstrated. The lens surface and bulk quality check performed using X-ray microtomography and atomic force microscopy reveal that the lenses are suitable for optical imaging. Furthermore, a smartphone microscope with $\sim 1.4-\mu \mathrm{m}$ resolution is developed using a self-assembly of a single high power fabricated lens and microaperture. The lenses have various potential applications, e.g., optofluidics, diagnostics, forensics, and surveillance. ๑ 2018 Society of Photo-Optical Instrumentation Engineers (SPIE) [DOI: 10.1117/1.JBO.23.2.025002]
\end{abstract}

Keywords: liquid mold; lens fabrication; curvature control; programmable curvature; smartphone microscope; microaperture fabrication.

Paper 170711R received Nov. 3, 2017; accepted for publication Jan. 18, 2018; published online Feb. 16, 2018.

\section{Introduction}

Low-cost handheld microscopes have a wide variety of applications, e.g., field deployable clinical diagnostics, ${ }^{1,2}$ forensics, ${ }^{3}$ environmental monitoring, ${ }^{4}$ surveillance, ${ }^{5}$ education, ${ }^{6}$ and biomedical research. ${ }^{7}$ Smartphones or webcams appended with low-cost miniature lenses have been shown to provide magnified images of samples with quality comparable with commercial microscopes. ${ }^{7-9}$ Further, these miniature lenses can be used in developing imaging systems for optical microsystem platforms, e.g., endoscopes. ${ }^{10}$

Various types of miniature lenses, e.g., ball lens, ${ }^{11,12}$ parabolic lens, ${ }^{9,13}$ plano-convex spherical lens, ${ }^{8}$ and reversed mobile phone camera lens, ${ }^{14}$ have been attached to smartphone cameras without hardware modifications. Thermal reflow, ${ }^{15}$ inkjet printing, ${ }^{16}$ photolithography, ${ }^{17}$ glass etching, ${ }^{18}$ and water-based molds ${ }^{19}$ are some of the established lens fabrication techniques. Most of these approaches require expensive equipment and complicated multistep processes for the fabrication of a master structure. Fluidic lenses, e.g., water droplets, offer the advantage of easily tunable focal lengths and naturally smooth surfaces. ${ }^{20}$ However, fluidic lenses require a provision for mechanical stability in the system and complex actuation mechanisms to manipulate the fluid.
In recent years, there has been a growing interest in a moldfree fabrication of solid lenses using droplets of viscous liquid PDMS (lPDMS). ${ }^{3,8,9,13}$ Mold-free techniques are less timeconsuming and cost-effective. Lee et al. ${ }^{13}$ reported a multistep mold-free approach using the naturally forming parabolic curvature of a hanging drop of $l$ PDMS. Lenses of increasing curvatures can be obtained by adding and curing $l$ PDMS repeatedly. Sung et al. ${ }^{8}$ showed that droplets of $l$ PDMS placed on a preheated smooth surface can be cured and used as lenses. The curvature of the resultant lenses can be changed by varying the volume of $l$ PDMS droplet and temperature of the hot surface. In both reported techniques, a controlled volume of a viscous solution ( $l$ PDMS) is required to be dispensed to ensure the reproducibility of the curvature of the fabricated lenses.

Recently, Nakakubo et al. ${ }^{21}$ reported a technique of lens fabrication using liquid metal mold. The study involves transfer of spherical surfaces prepared using the large surface tension force of liquid gallium to PDMS. After PDMS gets cured, gallium is suctioned out. Though the method is effective in producing lenses of a wide range of sizes and focal lengths, the reproducibility of the lenses has not been stressed. Seo et al. ${ }^{22}$ fabricated a variety of lenses by tuning the curvature of a flexible membrane with pressurized air. However, the technique may require a feedback mechanism to fabricate lenses reproducibly. 
In this report, we present a technique of lens fabrication using a hydrophilic solution as a liquid mold with programmable curvature. The curvature of the meniscus formed at the interface of lPDMS and the hydrophilic solution is controlled to obtain PDMS lenses (refractive index $\sim 1.4^{23}$ ) of a range of focal lengths. The science behind the curvature of the meniscus is described by the Young-Laplace equation. ${ }^{24}$ The novelty of our fabrication technique lies in its ability to use this science to fabricate solid optical lenses with off-the-shelf materials and equipment. A meniscus of $l$ PDMS is cured with in situ curvature control in a single PDMS curing step ( 20 min). When compared with the previous reported techniques on lens fabrication, our approach overcomes the drawback of the need to (1) dispense controlled volume of $l$ PDMS, ${ }^{3,8,9,13}$ (2) additively deposit $l$ PDMS for higher lens curvatures, ${ }^{13}$ and (3) align the PDMS dispensing unit with the base. ${ }^{3}$ This is achieved using a visual feedback system which includes a real-time magnified image of the meniscus formed at the $l$ PDMS/hydrophilic solution interface and a software-based meniscus guiding system. Further, we have developed a smartphone microscope using a single fabricated lens and demonstrated the ability of the system to image biological cells and tissue samples. The microscope is capable of differentiating between infected and healthy red blood cells in a malaria infected human blood smear visibly and using a designed color filter algorithm.

\section{Experimental Details}

\subsection{Equipment and Chemicals}

The $l$ PDMS/glycerol interface is formed at an aperture, which is cut in a $120-\mu$ m-thick transparent poly(ethylene terephthalate) (PET) sheet using Nd:YAG solid-state laser (F3w-40, YUCO optics, New York). The aperture can also be created using commercially available punching machines. The meniscus imaging system is built using Leica $8 \times$ objective $(\mathrm{NA}=0.18)$, a $10 \times$ wide-field microscope eye-piece $(23 \mathrm{~mm}$ diameter) and an iBall $^{\circledR}$ ROBO K20 webcam. A 1-ml BD Tuberculin syringe is used for pressure control. Glycerol is purchased from Merck (Mumbai, India). PDMS (Sylgard ${ }^{\circledR} 184$ silicone elastomer kit) is procured from Dow Corning (Mumbai, India). Xiaomi MI4 smartphone (13MP camera) and Carl Zeiss Axio Imager Z1 (conventional transmission microscope) are used in the study. The translational stage used to build the smartphone microscope was purchased from Holmarc, Kochi, India (Model: TS-65). The histopathology slide (thyroid gland tissue sample) is purchased from V. K. Scientific Industries (Mumbai, India). The malaria ( $P$. falciparum) infected human blood sample is cultured in Molecular Parasitology Lab, IIT Bombay.

\subsection{Lens Fabrication}

The block diagram in Fig. 1(a) describes the basic principle of lens fabrication. The system broadly consists of three components: an interface at two immiscible liquids, pressure control, and visual feedback. The curvature of the meniscus formed at the interface is tuned using the pressure control mechanism. The visual feedback assists in controlling the meniscus curvature to fabricate lenses of different curvatures reproducibly.

Figure 1(b) shows a schematic of the setup used for lens fabrication with the three components. The lid of the chamber is designed to have a transparent PET sheet with a circular aperture (A), where the $l$ PDMS/glycerol interface forms. The chamber is filled with $60 \%$ glycerol and covered with the lid without air bubbles trapped inside. IPDMS is prepared by mixing the monomer and the curing agent in the ratio of 10:1, and degassed. Since $l$ PDMS and glycerol are immiscible liquids, when IPDMS is poured on $\mathbf{A}$, a meniscus is formed at the interface of the two liquids. The meniscus imaging system $(\mathbf{S})$ provides a real-time magnified view of the meniscus, which is subsequently displayed on a computer monitor. This is used as a visual feedback to adjust the hydrostatic pressure in glycerol and vary the meniscus curvature. The hydrostatic pressure in glycerol is manually controlled using the syringe.

A hot air gun is used to cure $l \mathrm{PDMS}$ (at $\sim 80^{\circ} \mathrm{C}$ to $90^{\circ} \mathrm{C}$ for $\sim 20 \mathrm{~min}$ ). The lens fabrication is done in two steps. Step 1: in the first $10 \mathrm{~min}$, the meniscus curvature is kept at minimum till IPDMS gets partially cured and becomes elastic. Step 2: in the next $10 \mathrm{~min}$, the hydrostatic pressure in glycerol is adjusted to obtain the required curvature till $l$ PDMS gets completely cured. The detailed protocol of lens fabrication is described in Appendix A.1. The partial curing of $l$ PDMS prevents it from wetting the side of the PET sheet in contact with glycerol for higher meniscus curvatures. The cured PDMS is easily peeled off from the lid, resulting in a plano-convex PDMS lens [Fig. 1(c)]. The lens is rinsed with running deionized water and dried. The diameter of the aperture in the PET sheet determines the lens diameter. The two pedestals and a cover slip [Figs. 1(d) and 1(e)] are used to maintain a consistent lens base thickness $(\sim 1 \mathrm{~mm})$ across all the lenses fabricated using this setup. The imaging system $(\mathbf{S})$ was made of an $8 \times$ objective $(\mathrm{NA}=0.18)$ and a $10 \times$ wide-field eye-piece (23 mm diameter) [Fig. 1(f)]. The eye-piece was attached to a webcam with variable focus ( $5 \mathrm{~cm}$ to infinity) to image the meniscus. The distance between the objective and the eye-piece was adjusted to achieve a working distance of $\sim 25 \mathrm{~mm}$ to focus on the meniscus.

Glycerol is chosen as it has the advantage of not evaporating from the chamber when the setup is not in use. PET is used to fabricate the aperture as it has an optimum contact angle ( $\sim 77 \mathrm{deg}$ ) with $60 \%$ glycerol. This is to have lenses with a range of curvatures for a given lens diameter. The provision of a variable focus webcam helps in using the same meniscus imaging system $(\mathbf{S})$ for lenses of the size range considered in this study.

\subsubsection{Meniscus curvature control}

The reproducibility of the lens fabrication technique is ensured by the visual feedback and a meniscus guiding system. The meniscus guiding system defines guide points $\left(C_{k}\right)$ on the axis normal to the plane of $\mathbf{A}$. The circular aperture appears as an ellipse since $\mathbf{S}$ is placed at an angle with respect to the plane of A. As shown in Fig. 2(a), A lies in the POQ plane and $\mathrm{ON}$ is the axis normal to the plane. Plane $V$ is the viewing plane of $\mathbf{S}$, which is at an angle $\varphi$ with respect to $\mathrm{ON}$. A is projected as an ellipse on $V$ with $L_{\text {major }}$ and $L_{\text {minor }}$ as the length of the major and minor axes, respectively. $L_{\text {major }}$ is equal to the diameter of A. $L_{\text {minor }}$ is obtained from the live view of the meniscus. The tilt angle $(\varphi)$ of $\mathbf{S}$ is computed as

$\varphi=\sin ^{-1}\left(\frac{L_{\text {minor }}}{L_{\text {major }}}\right)$.

$C_{k}$ is a guide point on $\mathrm{ON}$ at a distance $x$ from the center $(O)$ of the aperture. The guide point seen from $\mathbf{S}$ will be the projection of $C_{k}$ on the line $\mathrm{ON}^{\prime}$ at a distance $x \cos \varphi$ from $O$. Five guide 


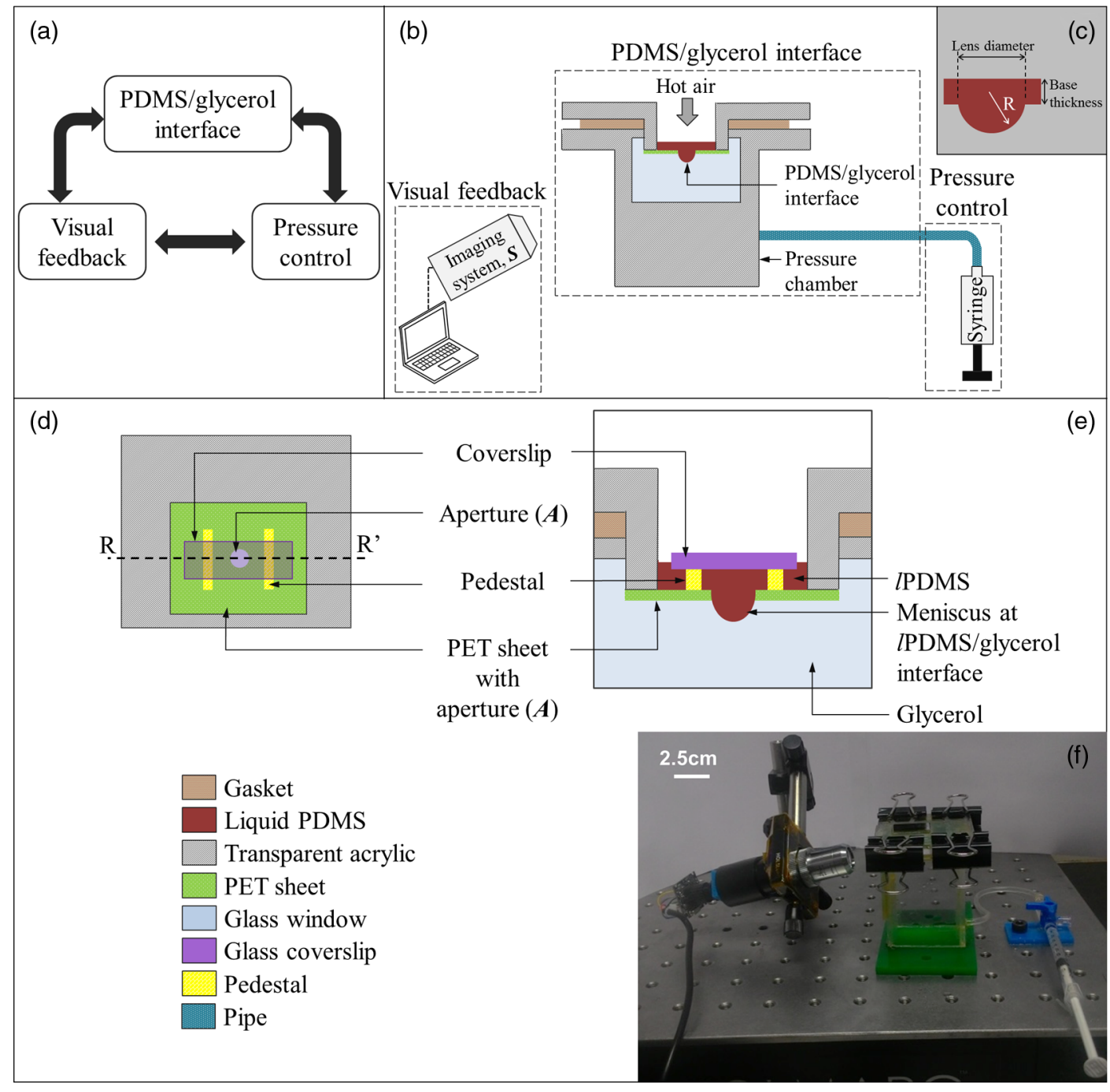

Fig. 1 Lens fabrication. (a) Basic principle, (b) schematic diagram showing various components of the setup, (c) lens terminology, (d) and (e) top view and side view (across RR') of the pressure chamber, respectively, showing different parts before IPDMS is poured, and (f) photo of the actual setup.
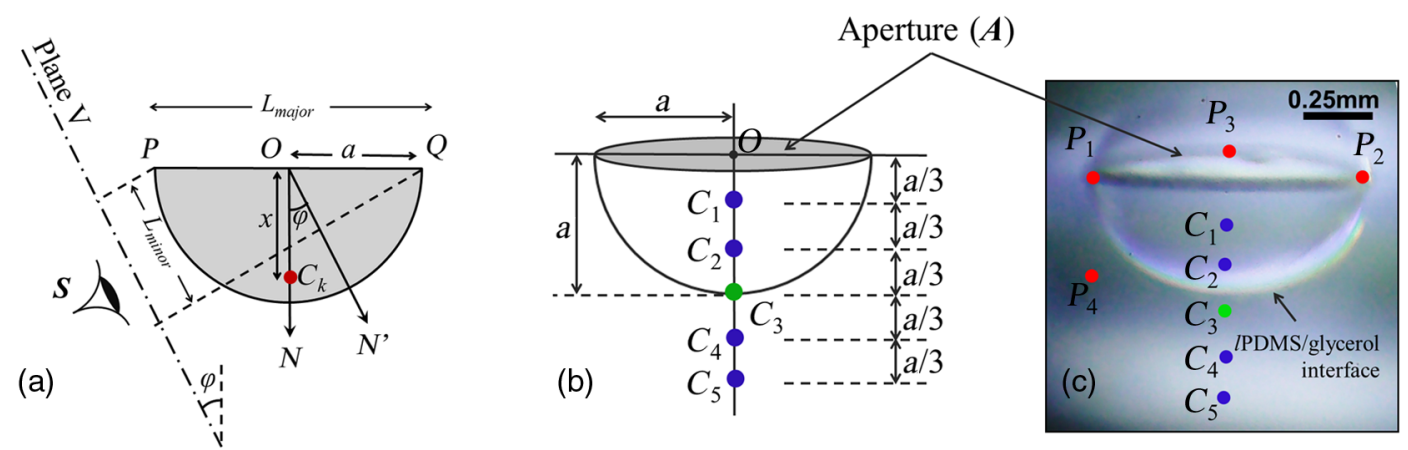

POQ is the plane of the aperture $(\boldsymbol{A})$

Fig. 2 Computation of guide points. (a) Projection of a guide point $C_{k}$ on the viewing plane (plane $V$ ) of $\mathbf{S}$. $C_{k}$ is on the axis normal to plane POQ. (b) Location of the guide points $C_{1}$ to $C_{5}$. (c) Live image of a 1 -mm-diameter meniscus displayed on the GUI with guide points superimposed. ${ }^{25} P_{1}$ to $P_{4}$ are points provided by the user (Fig. 3, Video 1). 
points $\left(C_{1}\right.$ to $\left.C_{5}\right)$ are defined by Eq. (2), where $k$ is the guide point number ranging from 1 to 5 and $a$ is the radius of $\mathbf{A}$ [Fig. 2(b)]

$x=\frac{k * a}{3}$.

In this study, the number and position of the guide points are arbitrarily chosen to demonstrate the control on curvature. There can be more guide points at different positions.

A graphic user interface (GUI) is developed in MATLAB $\mathrm{R} 2009 \mathrm{a}$ to (1) acquire and display the visual feedback from

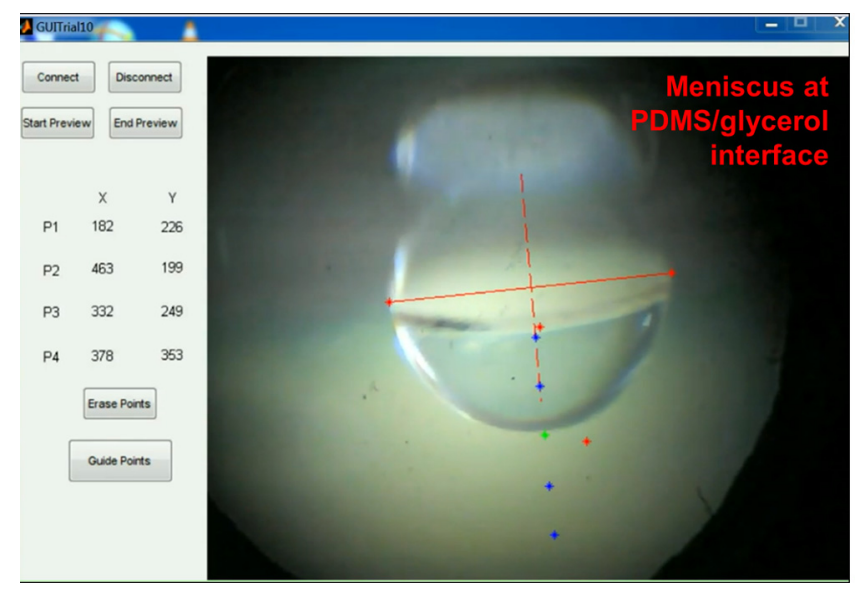

Fig. 3 The video shows the fabrication of a lens as seen by the graphic user interface of the meniscus guiding system. Meniscus at air/glycerol interface followed by PDMS/glycerol interface is shown. The red points indicate the user defined points. The blue and green points indicate the computed guide points. The green guide point specifically indicates that the lens fabricated using this guide point is hemispherical. In this video, the aperture diameter used is $2 \mathrm{~mm}$ and the lens produced is hemispherical. (Video 1, $\mathrm{mp} 4$, 6446 KB [URL: https://doi.org/10.1117/1.JBO.23.2.025002.1].)
S, (2) compute the guide points, and (3) superimpose them on the live image as shown in Fig. 2(c). In the GUI, the user defines the plane of $\mathbf{A}$ by selecting the four points $P_{1}$ to $P_{4}$. Subsequently, the GUI computes the guide points $C_{1}$ to $C_{5}$, and superimposes them on the visual feedback display. The user defined points are used to compute: ellipse major axis $\left(P_{1}\right.$ and $\left.P_{2}\right)$, ellipse minor axis $\left(P_{3}\right)$, and the direction of the meniscus with respect to the plane of $\mathbf{A}\left(P_{4}\right)$. Thus, the user should define a minimum of four points to describe the interface. In this study, PDMS lenses with different curvatures corresponding to the guide points are fabricated using two apertures (A) of diameters 1 and $5 \mathrm{~mm}$, respectively.

\subsection{Microaperture Fabrication}

Microaperture was fabricated using capillary encapsulation technique as reported by Cybulski et al. ${ }^{12}$ for glass ball lenses. This technique is low cost and allows self-assembly of the microaperture with the lens. We modified this technique for PDMS lenses (Fig. 4). A glass slide with PDMS ( 1-mm thick) was used to flatten a 1-mm-diameter PDMS lens [Fig. 4(a)]. The PDMS side of the glass slide was in contact with the curved side of the lens. A capillary was created between the two surfaces into which an opaque polymer solution was poured [Figs. 4(b) and 4(c)]. Once the opaque solution cures, the glass slide was removed. The lens gets back to its original shape due to the elastic nature of PDMS [Fig. 4(d)]. Thus, the microaperture was self-assembled with the lens in the resultant structure. The resultant aperture diameter was measured as $\sim 613 \mu \mathrm{m}$ [Fig. 4(e)].

\section{Results and Discussion}

When compared with the previous reports on lens fabrication,,$^{3,8,9,13}$ the presented technique has overcome the need to dispense a controlled volume of PDMS, and directs the focus to the pressure control mechanism and real-time visual feedback. In fact, the real-time visual feedback and the meniscus guiding (a)

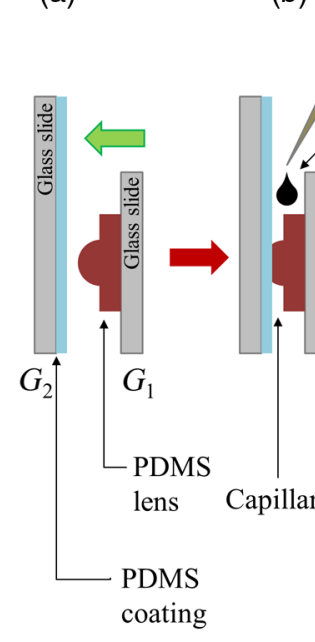

(c)

)

Micropipette

Plastic solution
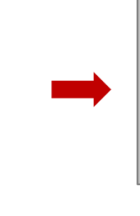

政 (d)

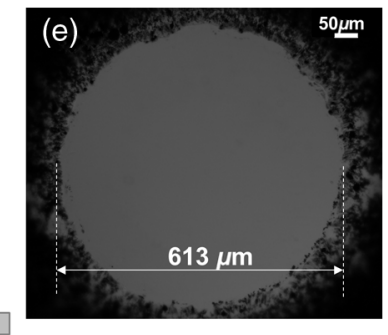

Fig. 4 Microaperture fabrication using capillary encapsulation technique. (a) Two glass slides $G_{1}$ and $G_{2}$ in parallel with a PDMS lens mounted on $G_{1}$. (b) Capillary formation due to lens flattening. (c) Encapsulation of the lens by the opaque plastic solution (Smooth-on Smooth-cast ONYX ${ }^{\circledR}$ ). (d) Final structure in which the microaperture is self-assembled on the lens after the opaque solution cures. $G_{1}$ and $G_{2}$ are separated and the lens resumes its original shape. (e) The fabricated microaperture under a transmission microscope. 

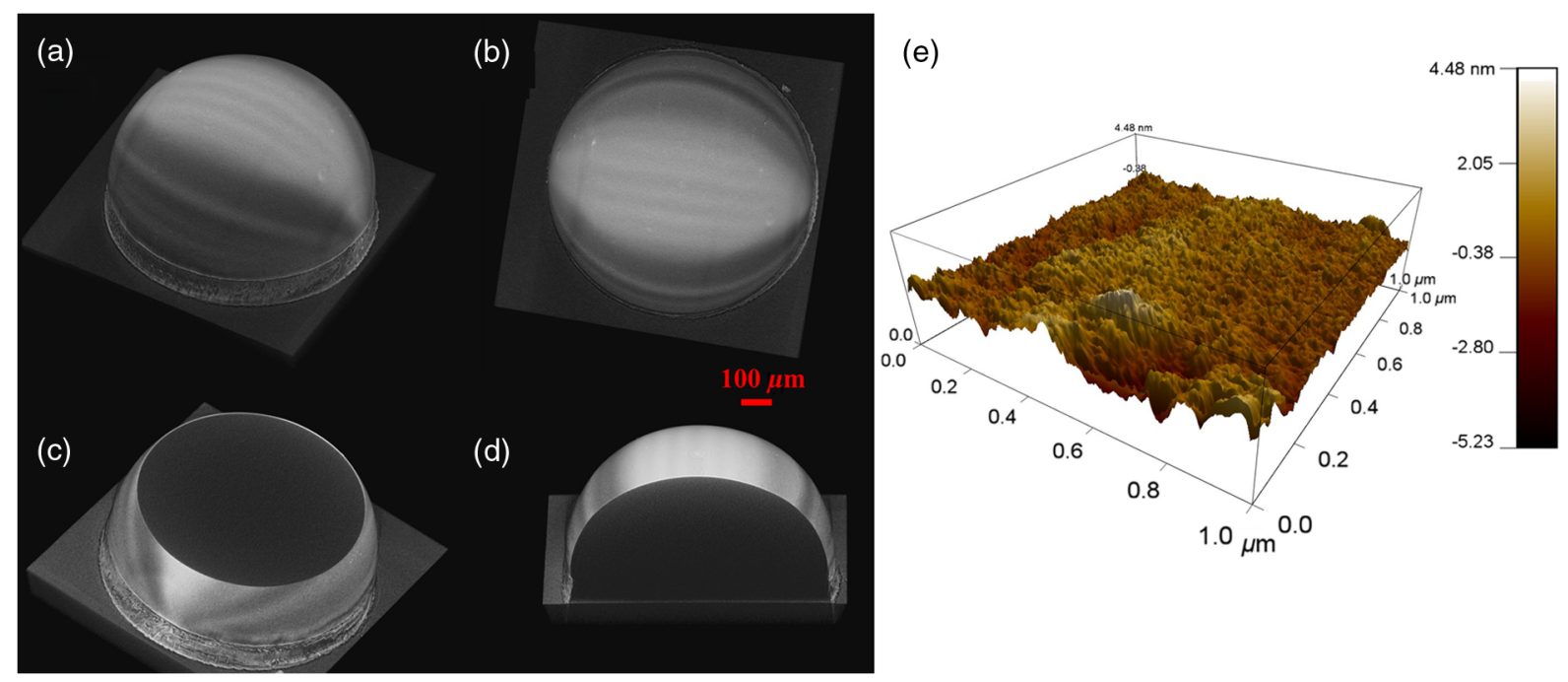

Fig. 5 The bulk and surface characterization of a 1-mm PDMS lens. (a-d) X-ray microtomography (microCT) images. (a) and (b) The side and top-view, respectively. (c) and (d) The lens at two different sectional planes (Fig. 6, Video 2). The images show that the lenses are nearly free of defects or ripples on the surface, and have no defects in the bulk. (e) AFM image across $1 \mu \mathrm{m} \times 1 \mu \mathrm{m}$ area on the lens surface. The root mean square surface roughness is measured as $628 \mathrm{pm}$.

system are designed to tune the curvature of the meniscus with ease and fine control by adjusting the pressure inside the chamber with a syringe.

\subsection{Lens Surface Roughness}

The bulk and surface characterization of the fabricated PDMS lenses is performed using X-ray microtomography (microCT) and atomic force microscopy (AFM) studies (Fig. 5). The microCT images demonstrate that (1) the lens surface is nearly free of defects or ripples [Figs. 5(a) and 5(b)], and (2) the bulk of

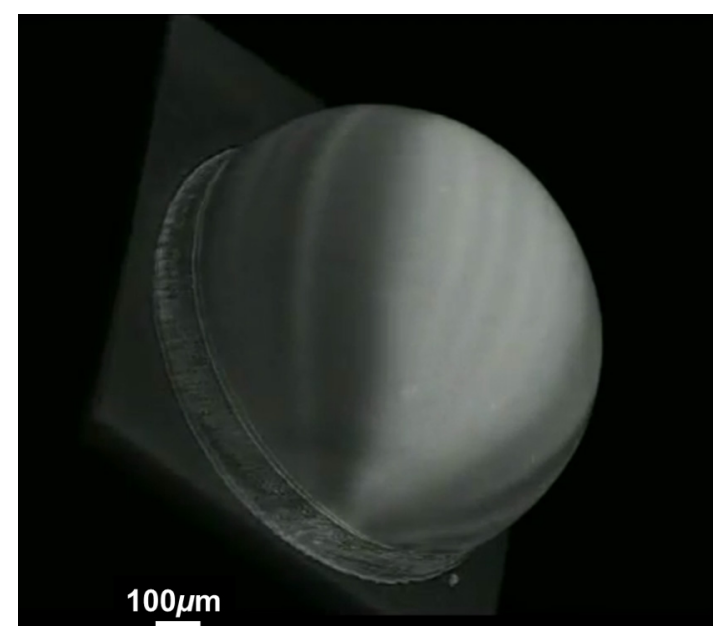

Fig. 6 MicroCT of a fabricated lens. The lens is fabricated using 1-mm-diameter aperture. The movie shows the side and top-view of the lens. Also, the bulk of the lens is shown across two different sectional planes. The microCT analysis shows that the fabricated lenses are nearly free of defects or ripples on the surface, and have no defects in the bulk (Video 2, mp4, $618 \mathrm{~KB}$ [URL: https:// doi.org/10.1117/1.JBO.23.2.025002.2].) the lens is also defect free [Figs. 5(c) and 5(d)]. The root mean square surface roughness is measured as $628 \mathrm{pm}$ using AFM [Fig. 5(e)]. This value is much less than $\frac{\lambda}{20}$ (assuming $\lambda=550 \mathrm{~nm}$ for white light illumination). The images demonstrate that the fabrication technique produces high quality, almost defect-free lenses that are suitable for optical imaging. The planar face of the fabricated lens is formed with the help of a glass coverslip of $\sim 2$-nm root mean square surface roughness.

\subsection{Characterization of Lens Radius of Curvature (R) and Focal Length (f)}

The fabricated PDMS lenses using 1- and 5-mm aperture diameters are characterized for radius of curvature and focal length as shown in Fig. 7. Figures 7(a) and 7(d) show the side-view of the lenses with various curvatures for 1 and $5 \mathrm{~mm}$ diameters, respectively. The nature of the lens profile is determined by fitting a circle to the side-view image of the lens using least squares approximation. It is observed that the circle fits the lens profile with $>99 \%$ approximation (Appendix A.2).

The plots of radius of curvature versus guide point are shown in Figs. 7(b) and 7(e) for 1- and 5-mm lens diameters, respectively. The experimental radius of curvature is estimated to be the radius of the circle that fits the lens profile in the side-view image. The theoretical radius of curvature is geometrically computed as in Eq. (3), where $a$ is the radius of $\mathbf{A}$ and $k$ is an integer ranging from 1 to 5 corresponding to the guide points $C_{1}$ to $C_{5}$, respectively,

$R=\frac{\left(k^{2}+9\right) * a}{6 k}$.

It is noticed from Fig. 7(b) that the experimental radius of curvature does not change significantly with the position of the guide point beyond $C_{3}$. The plot also shows that lenses are fabricated 
(a)

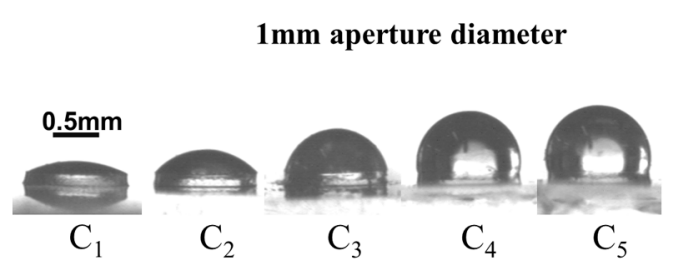

(d)

$\mathbf{5 m m}$ aperture diameter
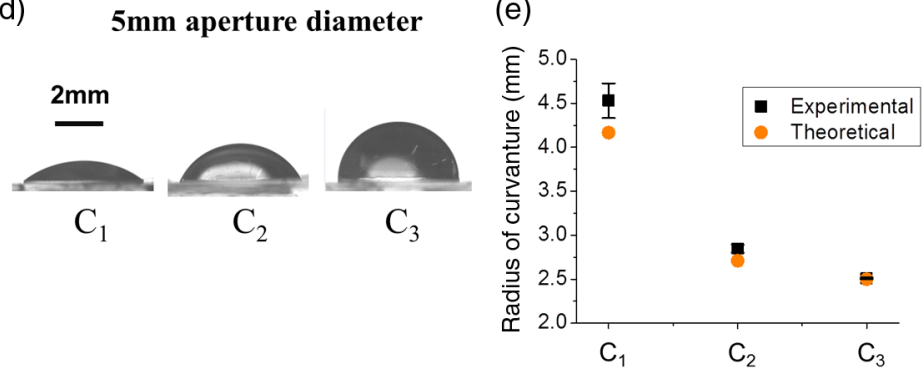

(b)
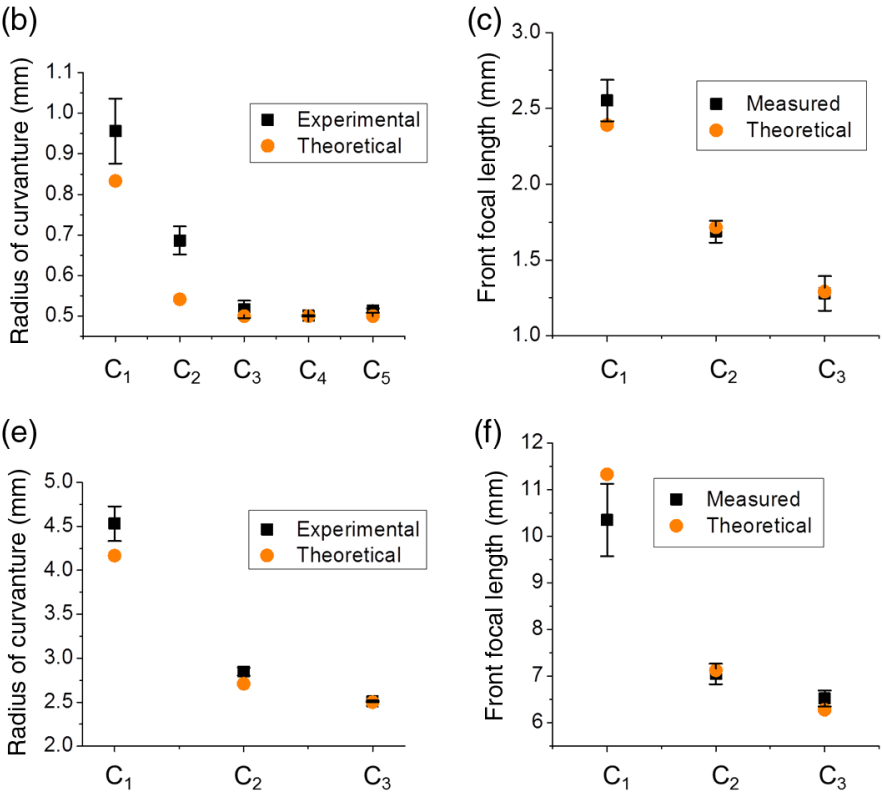

Fig. 7 Radius of curvature and focal length characterization of PDMS lenses fabricated using (a-c) $1 \mathrm{~mm}$ and (d-f) 5-mm aperture diameters. (a) and (d) The side-view images of the lenses. Plots (b,e) and (c, f) show the radius of curvature and focal length obtained for different guide points, respectively. The error bars represent the deviations across three lenses. A total of 24 lenses were fabricated to study the reproducibility of the fabrication technique.

with high reproducibility (up to $8 \%$ error) for higher lens curvatures $C_{3}, C_{4}$, and $C_{5}$. Similarly, the plot for radius of curvature versus guide point for 5-mm-diameter lens [Fig. 7(e)] shows that larger lenses of higher curvatures $\left(C_{3}\right)$ can be fabricated with even higher reproducibility $(0.8 \%$ error $)$.

In both the plots, the lower curvatures of the lenses $\left(C_{1}\right.$ and $C_{2}$ ) show larger deviation from theoretical estimates. This can be majorly attributed to (1) the tilt angle of the imaging system and (2) visual error. The visual error is induced during fabrication in (a) choosing $P_{1}, P_{2}$, and $P_{3}$, and (b) passing the meniscus through the desired guide point. The percentage error is calculated as $100 *\left(\frac{2 * \sigma}{\mu}\right)$, where $\mu$ and $\sigma$ are the mean and standard deviation, respectively, for experimental radius of curvature for a given guide point across three lenses.

Figures 7(c) and 7(f) show the front focal length obtained for different guide points for 1 - and 5-mm aperture diameters, respectively. The setup used for the focal length measurement is described in Appendix A.3. The theoretical values are computed using simplified Lens Maker's formula as in Eq. (4), where $R$ is the radius of curvature of the lens and $n$ is the refractive index of PDMS.

$f=\frac{R}{n-1}$.

The propagation of error from the measured radius of curvature is attributed to be the major factor for the variation in the measured focal length values. The plot shows $<4 \%$ deviation in measured and theoretical focal lengths for curvatures $C_{2}$ and $C_{3}$. The percentage deviation is calculated as $100 * \frac{\mid \text { Measured-Theoretical } \mid}{\text { Theoretical }}$.

\subsection{Development of a Smartphone Microscope (1smart)}

We developed a smartphone microscope $\left(\mathbf{I}_{\text {smart }}\right)$ by placing a single fabricated PDMS lens of high optical power externally to a smartphone [Fig. 8(a)]. The lens is fabricated using 1-mm aperture $\mathbf{A}$ diameter and guide point $C_{3}$ (focal length, $f=1.2 \mathrm{~mm}$ ). A 0.6 - $\mathrm{mm}$-diameter microaperture is selfassembled on the lens using the capillary encapsulation technique as described in Fig. 4. The presence of a microaperture prevents the peripheral rays from getting collected by the PDMS lens. Figure 8(b) shows the actual image of the portable 3-D printed prototype of $\mathbf{I}_{\text {smart }}$. The smartphone is placed on a fixed platform. A vertically movable translational stage with resolution of $0.01 \mathrm{~mm}$ is used for sample movement. A slip-on module is designed to house the self-assembly of a PDMS lens and a microaperture [Figs. 8(c) and 8(d)]. The intensity of illumination is varied using a potentiometer. The working distance of $\mathbf{I}_{\text {smart }}$ is measured to be $2.11 \mathrm{~mm}$.

The performance of $\mathbf{I}_{\text {smart }}$ is evaluated in comparison with a conventional microscope as shown in Fig. 9. Figures 9(a) and 9(b) show an image of a positive USAF 1951 resolution target acquired using $\mathbf{I}_{\text {smart }}$ and the conventional microscope, respectively. Figure 9(c) shows the normalized line intensity of Element 4 Group 8 from $\mathbf{I}_{\text {smart }}$ and the conventional microscope for comparison. The plot shows that line pairs of $1.38 \mu \mathrm{m}$ spacing can be separated by $\mathbf{I}_{\text {smart }}$. The fringe visibility $(V)$ is computed as $^{8}$

$V=\frac{I_{\max }-I_{\min }}{I_{\max }+I_{\min }}$

$I_{\max }$ and $I_{\min }$ are the maximum and minimum intensity, respectively, across a line passing through the fringe pattern. The 


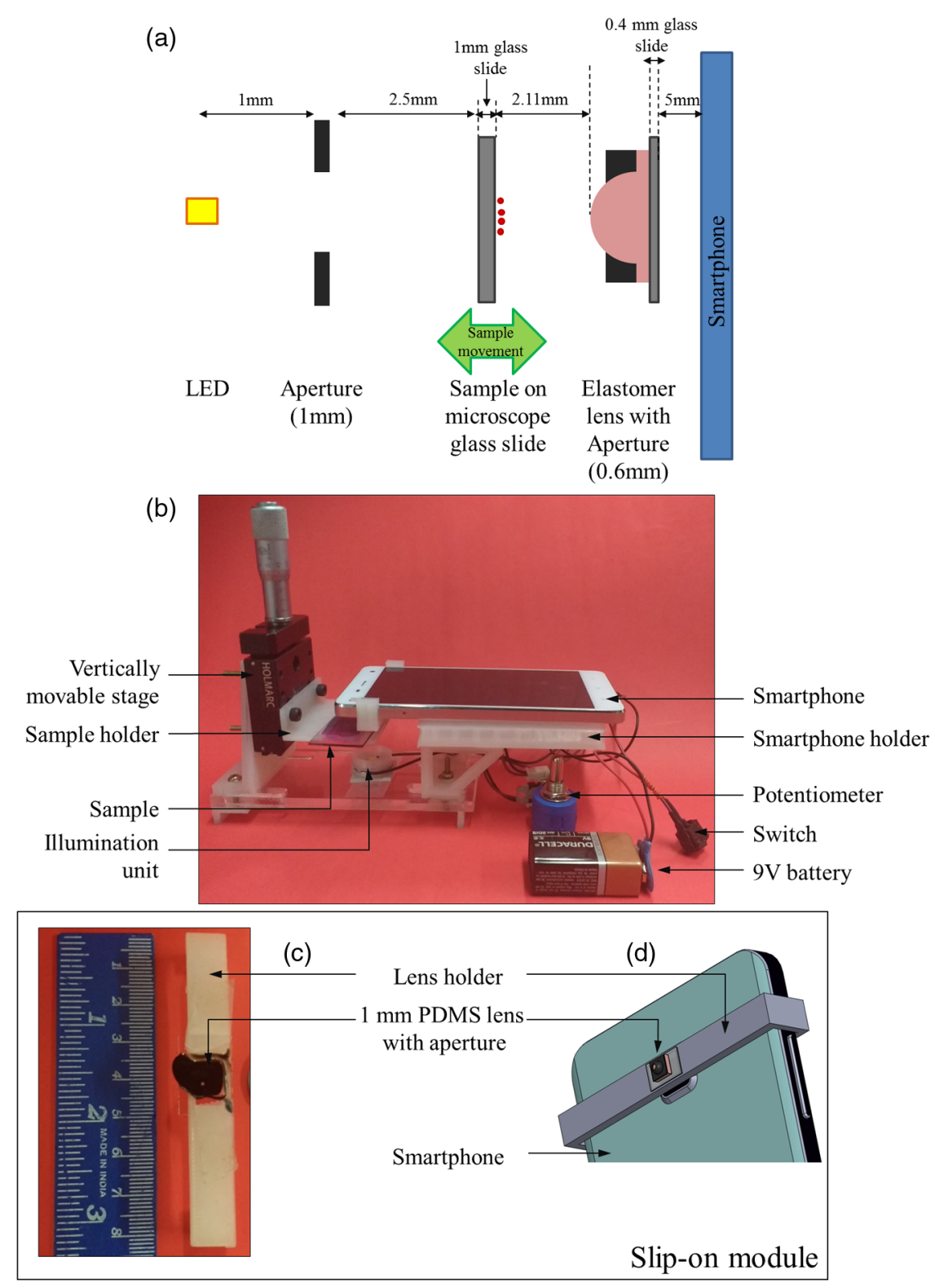

Fig. 8 Smartphone microscope $\left(\boldsymbol{I}_{\text {smart }}\right.$ ). (a) Schematic representation showing the various components. (b) Portable 3-D printed prototype. (c) and (d) The actual and schematic diagram of the slip-on module, respectively. The slip-on module houses the self-assembly of a PDMS lens and a microaperture.

intensities are mapped to integer values ranging between 0 and 255. The fringe visibility of Element 4 from the conventional microscope and $\mathbf{I}_{\text {smart }}$ is 0.37 and 0.05 , respectively. The resolution of $\mathbf{I}_{\text {smart }}$ is estimated as $1.4 \mu \mathrm{m}$.

The depth of field of $\mathbf{I}_{\text {smart }}$ is estimated as $45 \mu \mathrm{m}$ using the axial intensity profile of a gold pattern on quartz. ${ }^{26}$ The image of the gold pattern is shown in Fig. 9(e). The minimum intensity is obtained across line $\mathrm{AA}^{\prime}$ for planes below and above the best focused plane. Figure $9(\mathrm{~d})$ shows the normalized minimum intensity for each plane versus distance of the plane from the best focused plane. The depth of field is computed as the distance within which the change in the minimum intensity is $<20 \%$ of its value at the best focused plane. The field of view of the system is estimated as $\sim 200-\mu$ m diameter by imaging a standard hemocytometer glass slide [Fig. 9(f)]. ${ }^{7}$

\subsection{Biological Sample Imaging}

In this section, the potential of $\mathbf{I}_{\text {smart }}$ to image biological samples is demonstrated and compared with a conventional microscope (Fig. 10). Image quality functions are computed in MATLAB R2015a using images of a thyroid tissue sample acquired using a conventional microscope as the reference image [Fig. 10(a)], and $\mathbf{I}_{\text {smart }}$ as the test image [Fig. 10(b)]. The peak signal-to-noise ratio (PSNR) and structural similarity index (SSIM) are computed as $19.04 \mathrm{~dB}$ and 0.87 , respectively. The value of $20 \mathrm{~dB}$ of PSNR is considered as recognizable with respect to the reference image. ${ }^{27}$ SSIM ranges between 0 and 1 , where 1 is for high similarity between the test and reference images. ${ }^{28}$ The values of the image quality metrics show that the images acquired by $\mathbf{I}_{\text {smart }}$ have similarity to the images from a conventional microscope. 

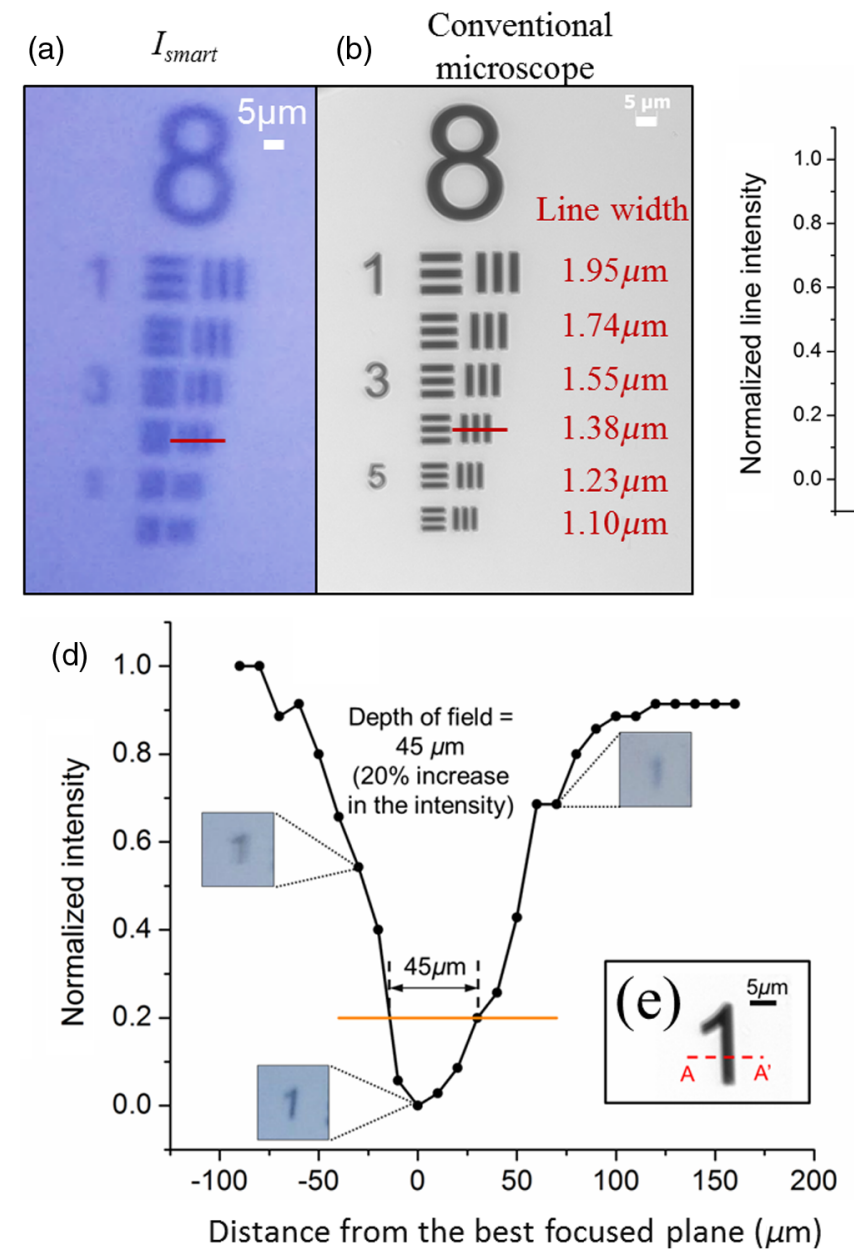

(c) Group 8, Element 4
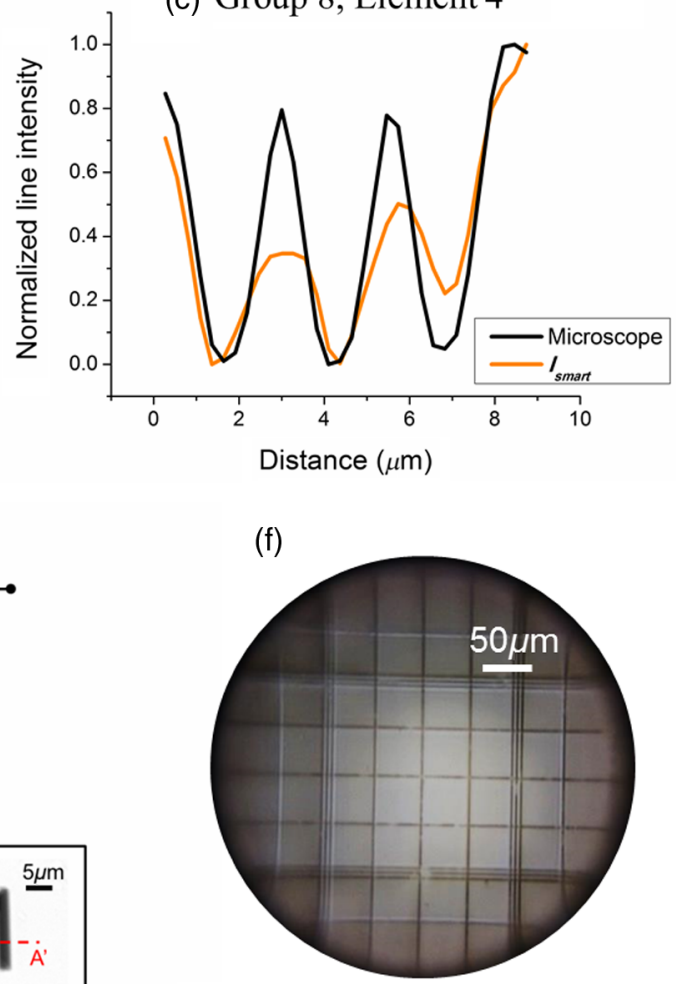

Field of view: $50 \mu \mathrm{m} \times 4=200 \mu \mathrm{m}$

Fig. 9 Performance metrics of $I_{\text {smart }}$ Images of a positive USAF 1951 resolution test target (Group 8) acquired by (a) $I_{\text {smart }}$ and (b) a conventional microscope ( $50 \times$ objective). (c) Comparison of the normalized line intensity across Element 4 for images acquired from $I_{\text {smart }}$ and microscope. The resolution of $I_{\text {smart }}$ is estimated as $1.4 \mu \mathrm{m}$. (d) Plot showing the minimum intensity computed across line $\mathrm{AA}^{\prime}$ for images below and above the best focused plane. The distances are normalized with respect to the best focused plane. The depth of field is computed as the distance within which the change in the minimum intensity is $<20 \%$ of its value at the best focused plane. (e) Line $A A^{\prime}$ across a gold pattern on quartz wafer. (f) Image of a hemocytometer glass slide acquired using $I_{\text {smart }}$. The field of view of $I_{\text {smart }}$ is estimated as $\sim 200-\mu \mathrm{m}$ diameter.

Figure 10(c) shows the malaria (P. falciparum) infected cells in a stained human blood smear (Field's stain) which can also be seen in the image acquired by $\mathbf{I}_{\text {smart }}$ [Fig. 10(d)]. Further, the image acquired by $\mathbf{I}_{\text {smart }}$ is subjected to an image processing algorithm (Appendix A.4) to locate the infected cells in a given image. A color filter algorithm is designed to indicate the malaria infected cells with black spots. As can be seen from Fig. 10(e), the algorithm is able to identify the infected cells clearly. Thus, we have demonstrated $\mathbf{I}_{\text {smart }}$ to be a potential affordable microscope capable of acquiring images of pathological samples.

\section{Conclusion}

In summary, we report a technique to reproducibly fabricate cheap lenses $(<0.01 \mathrm{USD})$ that are optically powerful enough to be used for developing simple, smartphone-based microscopes. The novelty lies in fabricating lenses with variable curvatures reproducibly using off-the-shelf materials within $20 \mathrm{~min}$. The visual assisted, lens curvature control is achieved during fabrication by varying the curvature of the interface of two immiscible liquids. To the best of our knowledge, this is the first time that the lens curvature control is achieved using a feedback mechanism. The visual feedback along with the meniscus guiding system is simple to implement and does not require any kind of calibration with the lens size or curvature. The technique offers the advantage of being able to fabricate lenses of a range of sizes and focal lengths. Among the fabricated lenses, the focal length ranged from 1.2 to $11 \mathrm{~mm}$ with $<8 \%$ variation in radius of curvature. This implies high reproducibility. Further, a technique to self-assemble a microaperture on the fabricated lens is shown. A smartphone transformed into a microscope using this assembly is capable of imaging biological cells and tissue samples. Such low-cost lenses and microscopes can have potential applications in clinical diagnostics, biomedical research, life-science education, surveillance, and designing low-cost optical microsystems, e.g., endoscopes, otoscopes, and dental microscopes. 

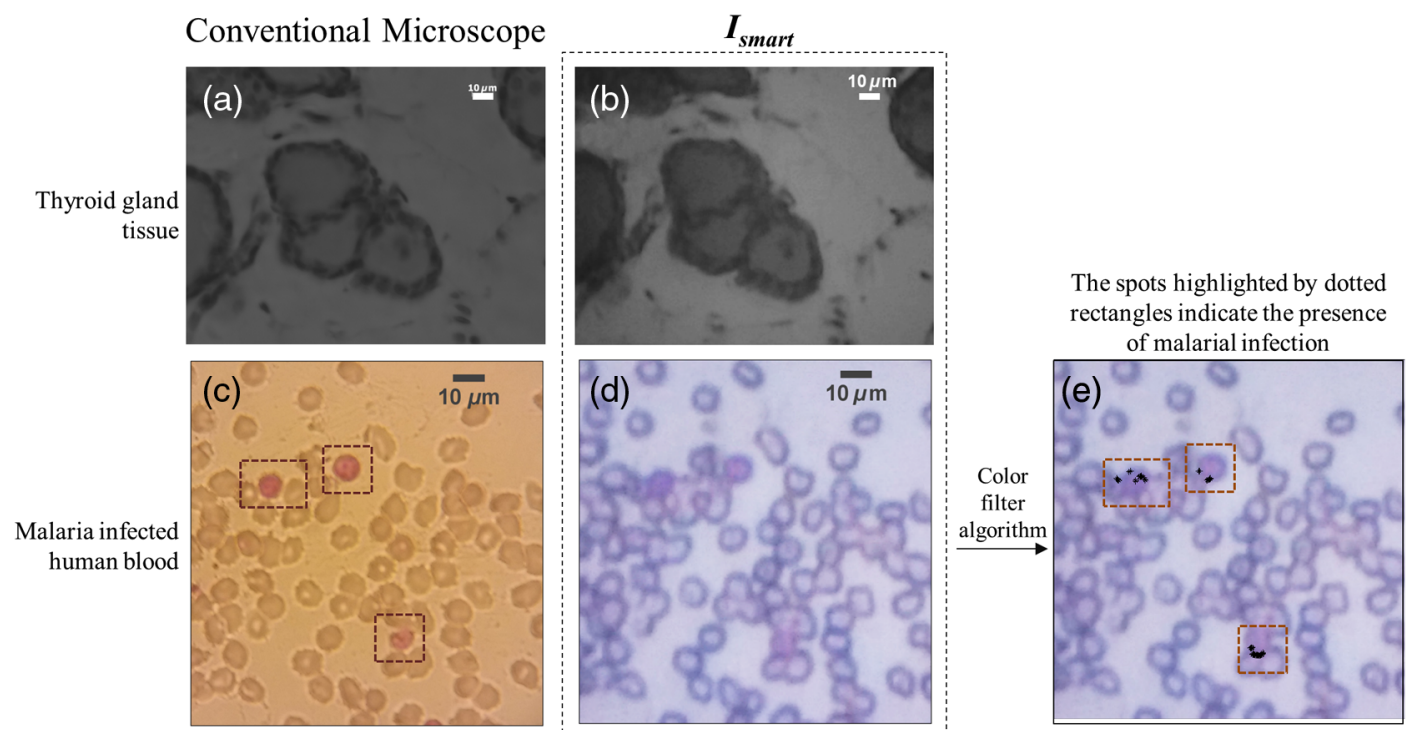

Fig. 10 Biological sample imaging. The images of thyroid gland tissue sample are acquired by (a) monochrome CCD camera mounted on a conventional microscope, and (b) $I_{\text {smart }}$ (image converted to grayscale). The images malaria ( $P$. falciparum) infected human blood smear stained with field's stain are acquired using (c) smartphone placed at the eye-piece of the conventional microscope, and (d) $I_{\text {smart }}$. The conventional microscope was fitted with a $50 \times$ objective for both the samples. The infected cells are highlighted by dotted rectangles. (e) The spots (highlighted by dotted rectangles) computed using the color filter algorithm show the presence of malarial infected cells in the sample.

\section{Appendix}

\section{A.1 Protocol for Lens Fabrication}

The two steps of lens fabrication are divided in three phases as described as follows:

- In phase 1 [Fig. 11(a)], an interface of liquid PDMS and glycerol is formed. The setup is at room temperature $\left(27^{\circ} \mathrm{C}\right)$. The pressure inside the chamber is controlled to have a flat meniscus curvature at the interface.

- In phase 2 [Fig. 11(b)], the hot air gun is switched on to cure liquid PDMS at $\sim 80^{\circ} \mathrm{C}$ to $90^{\circ} \mathrm{C}$. At this phase, the meniscus curvature is kept at minimum till PDMS gets partially cured.

- In phase 3 [Fig. 11(c)], the hot air gun is still switched on to maintain the temperature of the system at $\sim 80^{\circ} \mathrm{C}$ to $90^{\circ} \mathrm{C}$. At this phase, the pressure in glycerol is reduced to increase the meniscus curvature at the PDMS/ glycerol interface to fabricate the lens with desired curvature.

\section{A.2 Lens Profile Determination}

The fabricated lenses appear to be spherical. However, this can be statistically proved by fitting circle along the lens profile using least square approximation. Figure 12(a) shows the flowchart of the processes involved in the analysis. The analysis is done using MATLAB R2009a.

Figure 12(b) shows the side-view of the lens. First, the image is converted to black and white [Fig. 12(c)]. The data-points of
Phase 1: Temperature $=27^{\circ} \mathrm{C}$

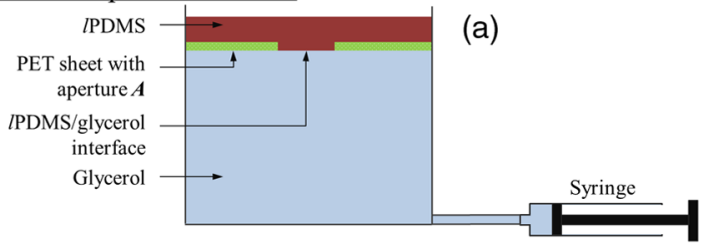

Phase 2: Temperature $=80^{\circ} \mathrm{C}$

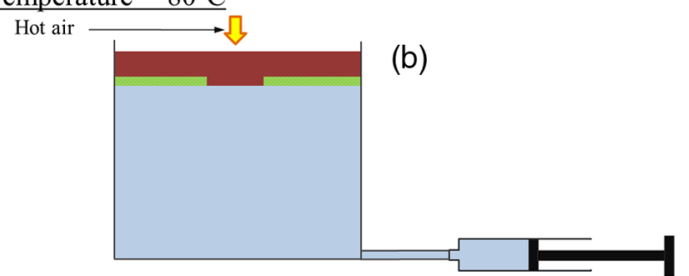

Phase 3: Temperature $=80^{\circ} \mathrm{C}$, meniscus curvature increase

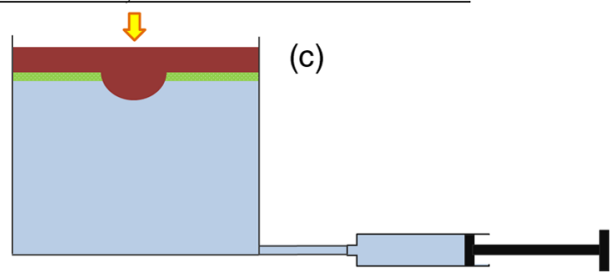

Fig. 11 Protocol for lens fabrication. (a) Phase 1: an interface of liquid $\mathrm{PDMS} / \mathrm{glycerol}$ is formed at the aperture at room temperature. The pressure inside the chamber is controlled to have a flat meniscus curvature at the interface. (b) Phase 2: PDMS is partially cured and becomes elastic. The meniscus curvature is maintained flat. (c) Phase 3 : the pressure inside the chamber is reduced to attain the desired meniscus curvature. PDMS is completely cured at this meniscus curvature. 


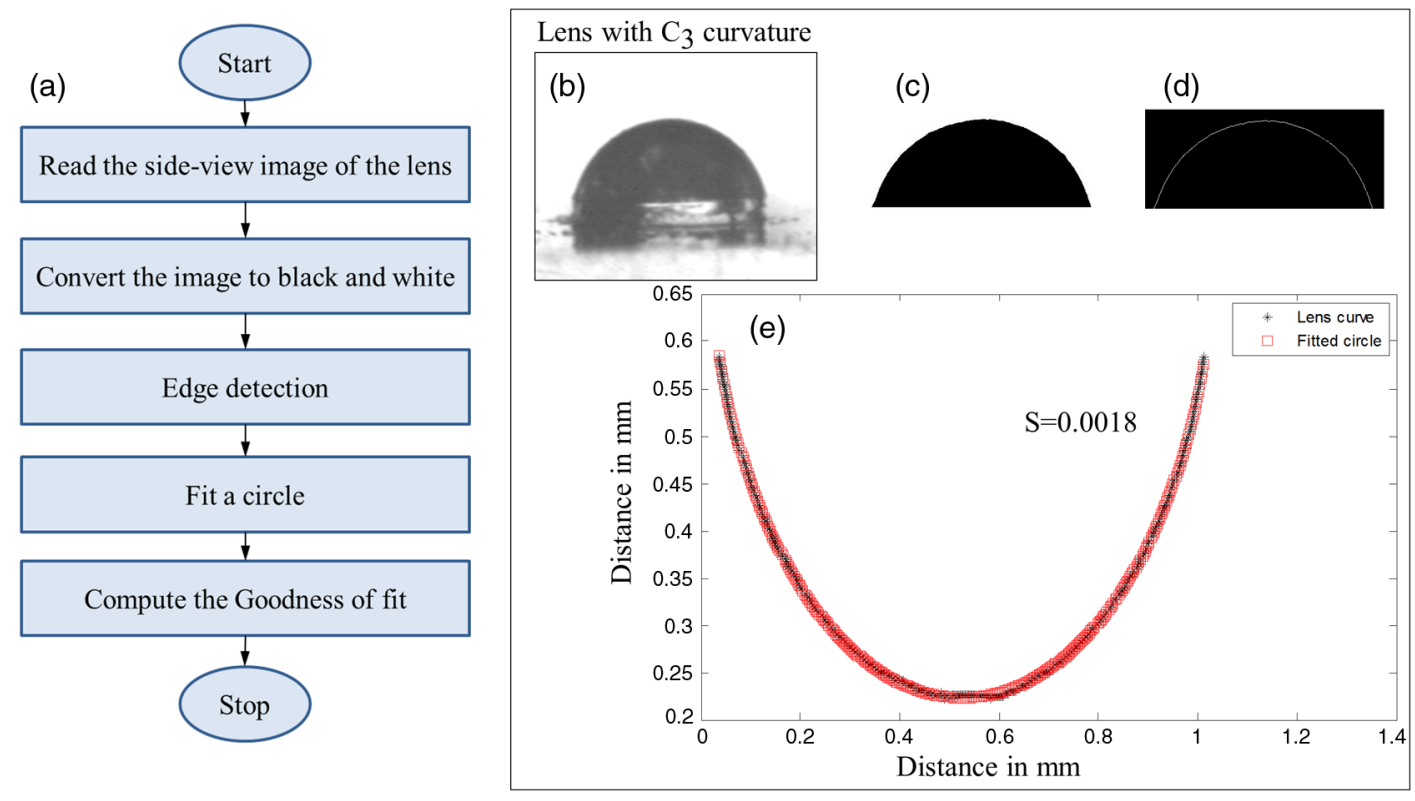

Fig. 12 Determination of the nature of lens profile. (a) Flowchart that includes (1) the data-points extraction, (2) circle fitting, and (3) computation of goodness of fit. (b) Side-view image of the lens. (c) A black and white image of the lens. (d) Data-points of the lens profile extracted from the image. (e) Fitting a circle along the extracted data-points. The goodness of fit used in this analysis is standard error of estimate $(S)$. For the given image, $S=0.0018$.

the lens profile are extracted from the black and white images of the lens by edge detection [Fig. 12(d)]. A circle is fit along the extracted data-points [Fig. 12(e)].

The goodness of fit is determined by standard error of estimate " $S$." In this analysis, R-squared is not applicable as it involves nonlinear regression. For the given example lens, $S=0.0018$. This indicates that the average distance of the data points from the fitted curve is $0.0018 \mathrm{~mm}=1.8 \mu \mathrm{m}$.

For a good approximation, $95 \%$ of the observations should fall within $\pm 2 * S$ of the regression curve. In all the lenses fabricated, this percentage value " $P$ " is $>99 \%$ (Fig. 13). Hence, this analysis shows that the profile of the fabricated lenses can be approximated as spherical.

\section{A.3 Focal Length Measurement Setup}

Figure 14 describes the setup used to measure the focal length of the fabricated PDMS lenses. An imaging system was developed using a $10 \times$ objective lens $(\mathrm{NA}=0.25)$, standard focusing lens

\section{(a) Aperture diameter $=\mathbf{1} \mathbf{~ m m}$}

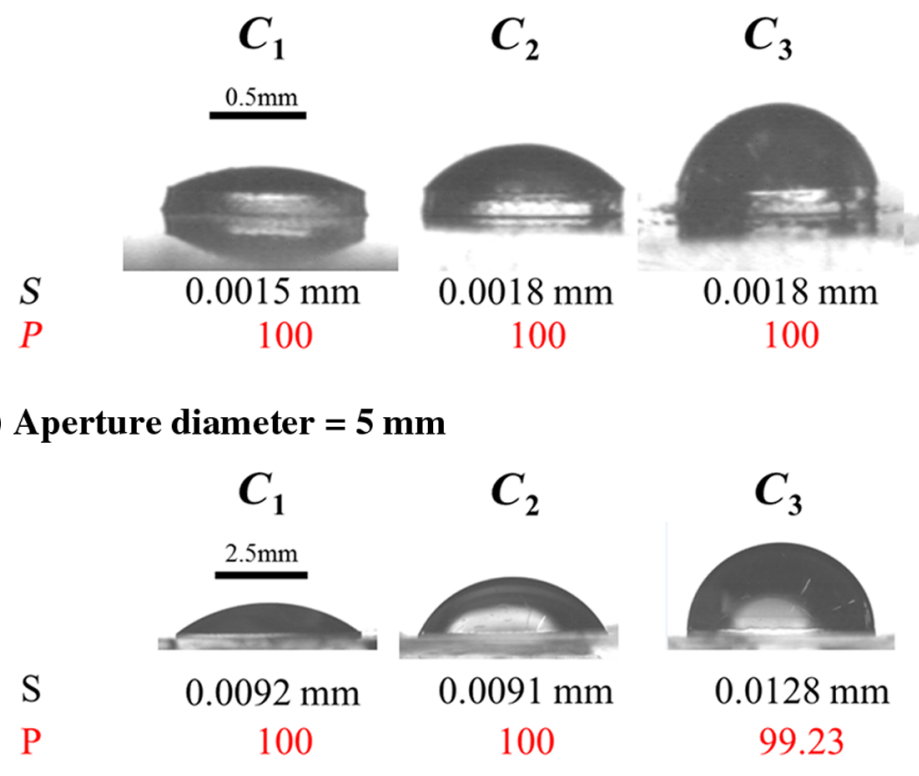

Fig. 13 Standard error of estimate " $S$ " and percentage of observations " $P$ " falling within $\pm 2 \times S$ of the fitted circle. The aperture diameter used for lens fabrication is (a) $1 \mathrm{~mm}$ and (b) $5 \mathrm{~mm}$. 


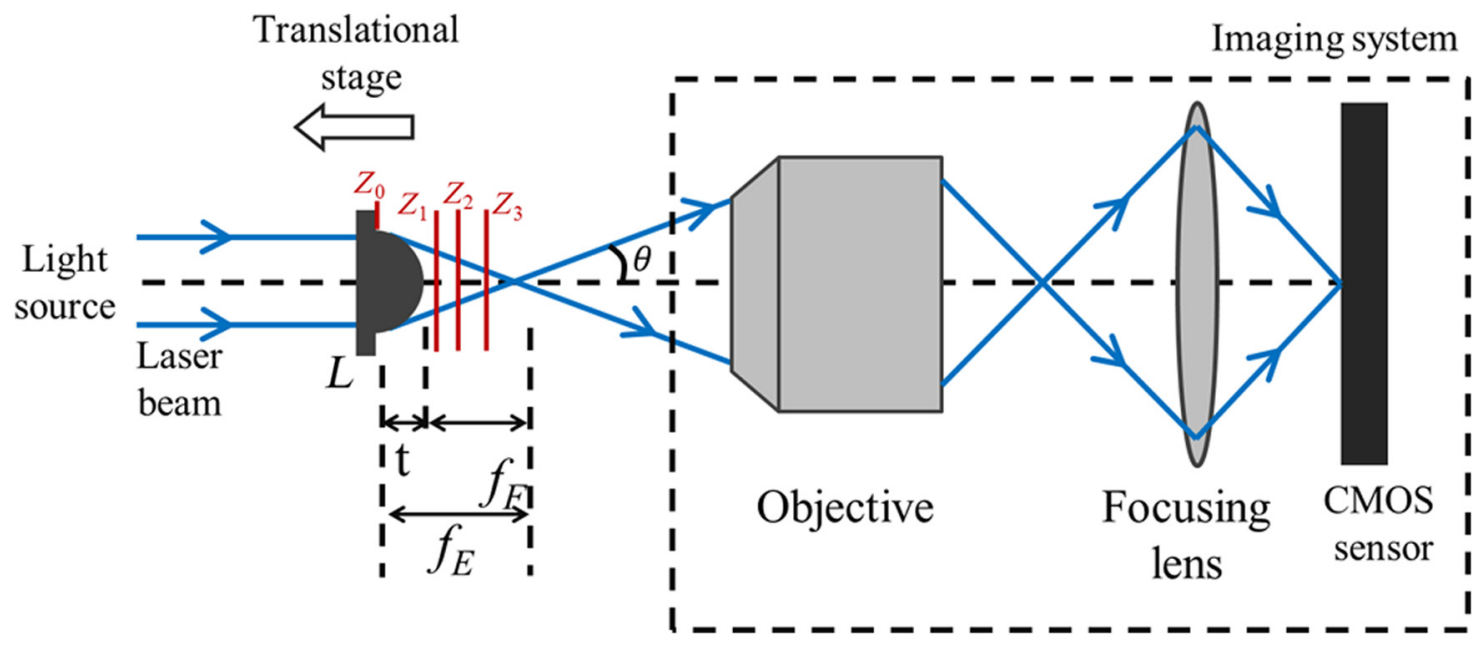

Fig. 14 Setup used to measure the focal length of the fabricated PDMS lens $(L)$. Planes $Z_{0}, Z_{1}, Z_{2}$, and $Z_{3}$ are cross-sectional planes across the converging beam. $Z_{0}$ is the plane across the base of the PDMS lens $(L)$.
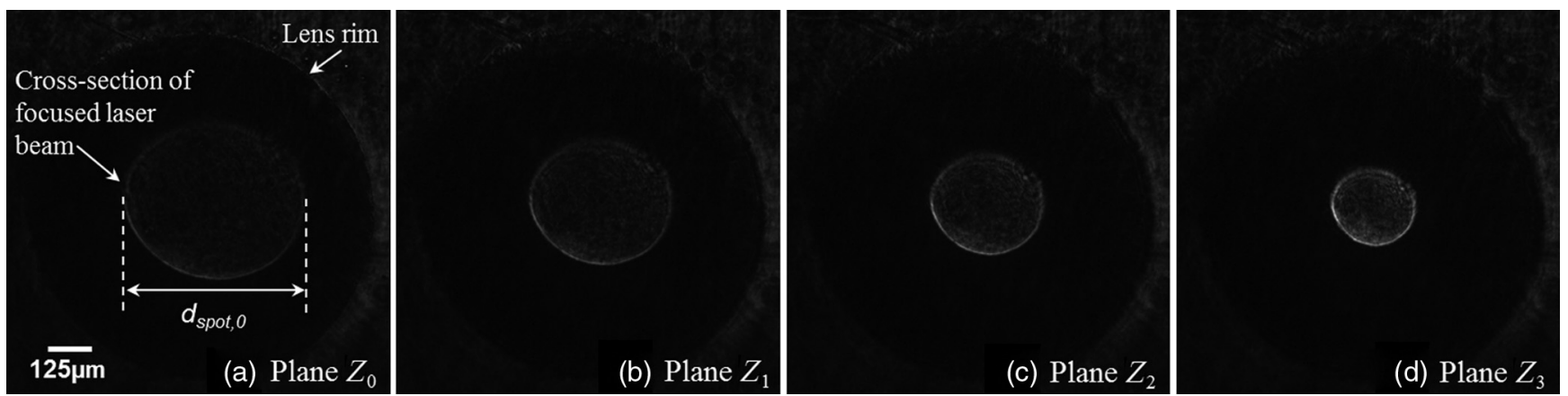

Fig. 15 Images acquired using the imaging system at (a) plane $Z_{0}$, (b) plane $Z_{1}$, (c) plane $Z_{2}$, and (d) plane $Z_{3}$.

(diameter $=25 \mathrm{~mm}$, focal length $=40 \mathrm{~mm})$, and CMOS camera (DCC1545M, Thorlabs, New Jersey). PDMS lens $(L)$ was placed on a calibrated manually actuated translational stage (TS-65, Holmarc, Kochi, India) with a maximum travel range of $15 \mathrm{~mm}$ and resolution of $0.01 \mathrm{~mm}$.

Initially, $L$ was illuminated with white LED as light source. Plane $Z_{0}$ (plane of the base of the lens) was brought to the focus of the imaging system using the translational stage. At this position, the LED was switched off and a collimated beam of red laser (wave length $=660 \mathrm{~nm}$ ) was switched on. The diameter of the spot formed by $L$ in the image was measured to be $d_{\text {spot, } 0}$ [Fig. 15(a)]. Planes $Z_{1}, Z_{2}$, and $Z_{3}$ are sequentially brought to the focus by moving $L$ away from the imaging system. For each of the planes, a cross-sectional image of the converging laser beam was acquired [Figs. 15(b)-15(d)].

The diameter of the beam spot and the distance moved by the translational stage for each of the images were used to determine the angle of convergence $(\theta)$ of the cone formed by the converging beam. The effective focal length $\left(f_{E}\right)$ is computed as

$f_{E}=\frac{d_{\mathrm{spot}, 0}}{2 * \tan \theta}$.
The curved thickness $(t)$ of PDMS lens $(L)$ is computed as $t=R_{m}-\sqrt{R_{m}^{2}-a^{2}}$,

where $R_{m}$ is the measured radius of curvature of the lens and $a$ is the radius of the base of the lens. The front focal length $\left(f_{F}\right)$ is defined as

$f_{F}=f_{E}-t$.

\section{A.4 Algorithm for Color Filter}

Figure 16 describes the steps involved in the color filter algorithm used to detect malarial infection in an image acquired by $\mathbf{I}_{\text {smart }}$.

Steps:

1. Background subtraction was done in ImageJ $1.50 \mathrm{i}$ with settings as shown in Fig. 17 to enhance the color of the infected cells.

2. The intensity corresponding to the color of the infected cells is determined using MATLAB R2015a. The 


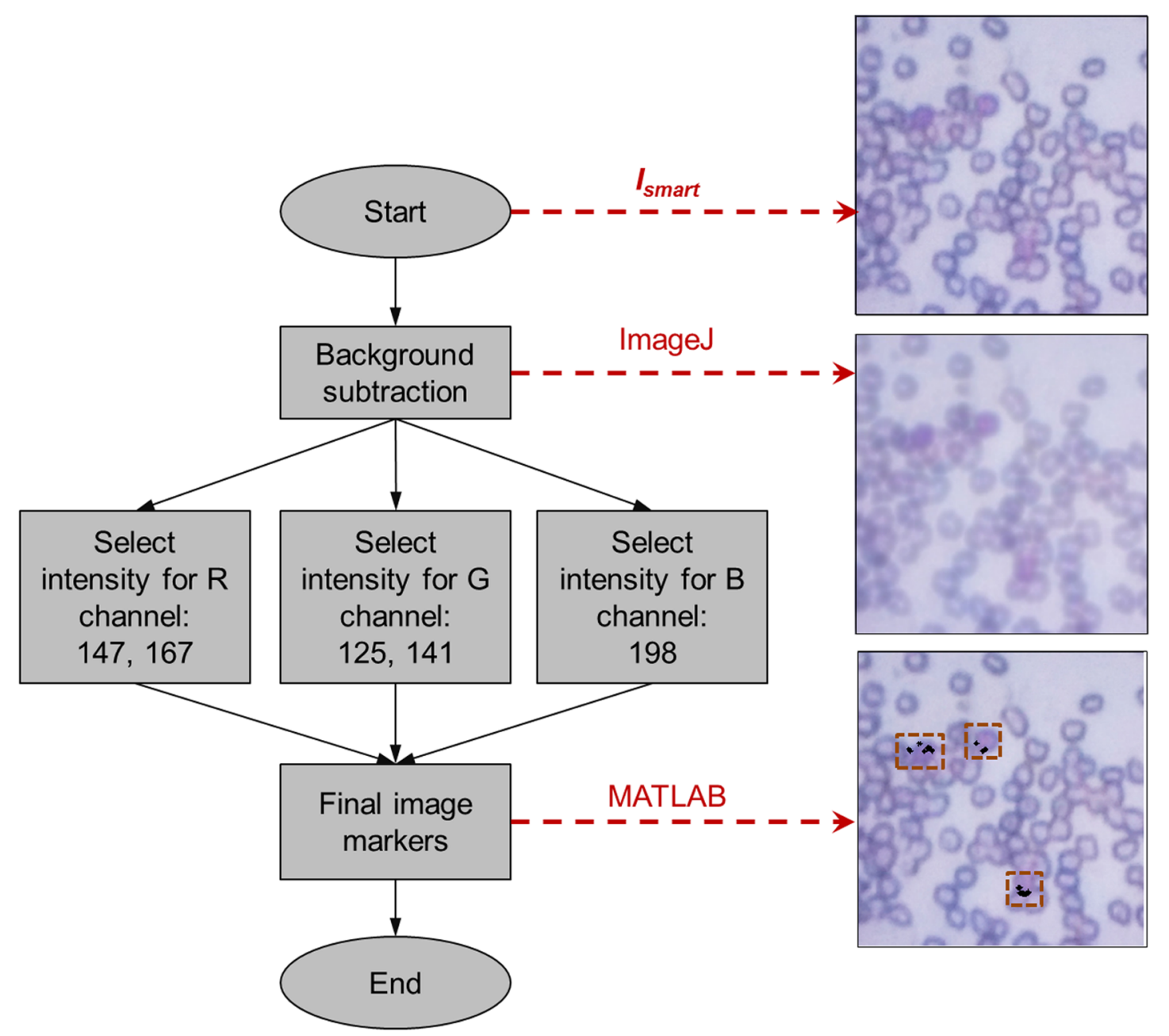

Fig. 16 Flowchart of the color filter algorithm used to detect malarial infection in an image acquired by Ismart.

intensity values for each of the RGB channels are determined by plotting and comparing the histograms of the images acquired from (a) background subtracted image and (b) manually isolated infected cells (Fig. 18). Figure 19 shows the histogram of

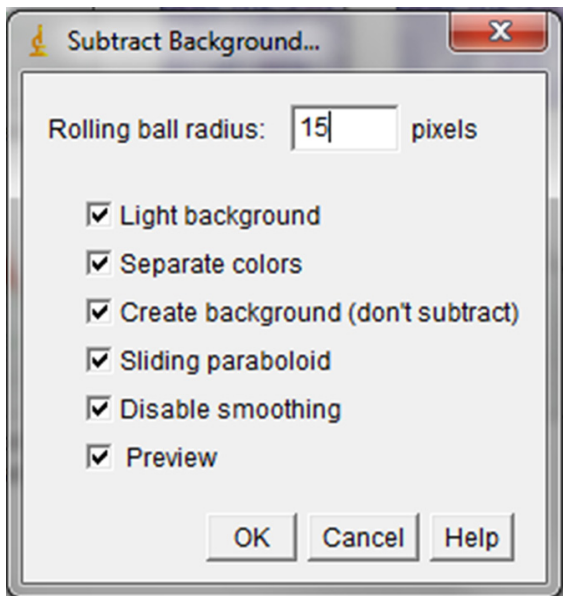

Fig. 17 Settings of the background subtraction tool in ImageJ 1.50i. intensity for RGB channels: (a) red, (b) green, and (c) blue channel. The intensity values at the peaks are considered to be the RGB values for identifying the malarial infected cells in the given image acquired by $\mathbf{I}_{\text {smart }}$.

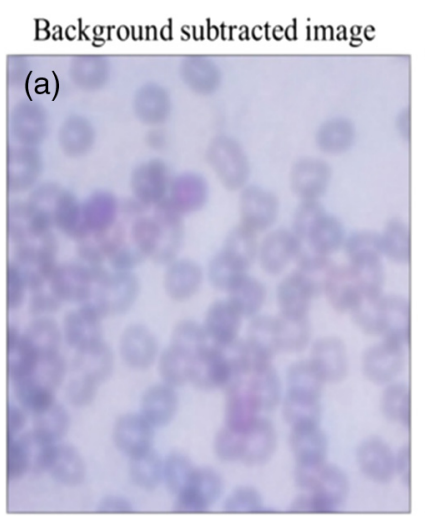

Infected cells isolated from (a)

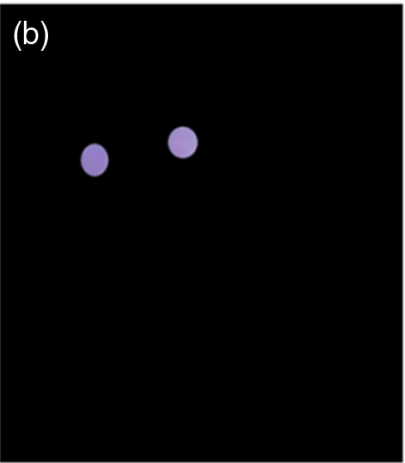

Fig. 18 Images considered for plotting histogram of intensity from (a) background subtracted image and (b) manually isolated infected cells from (a). 

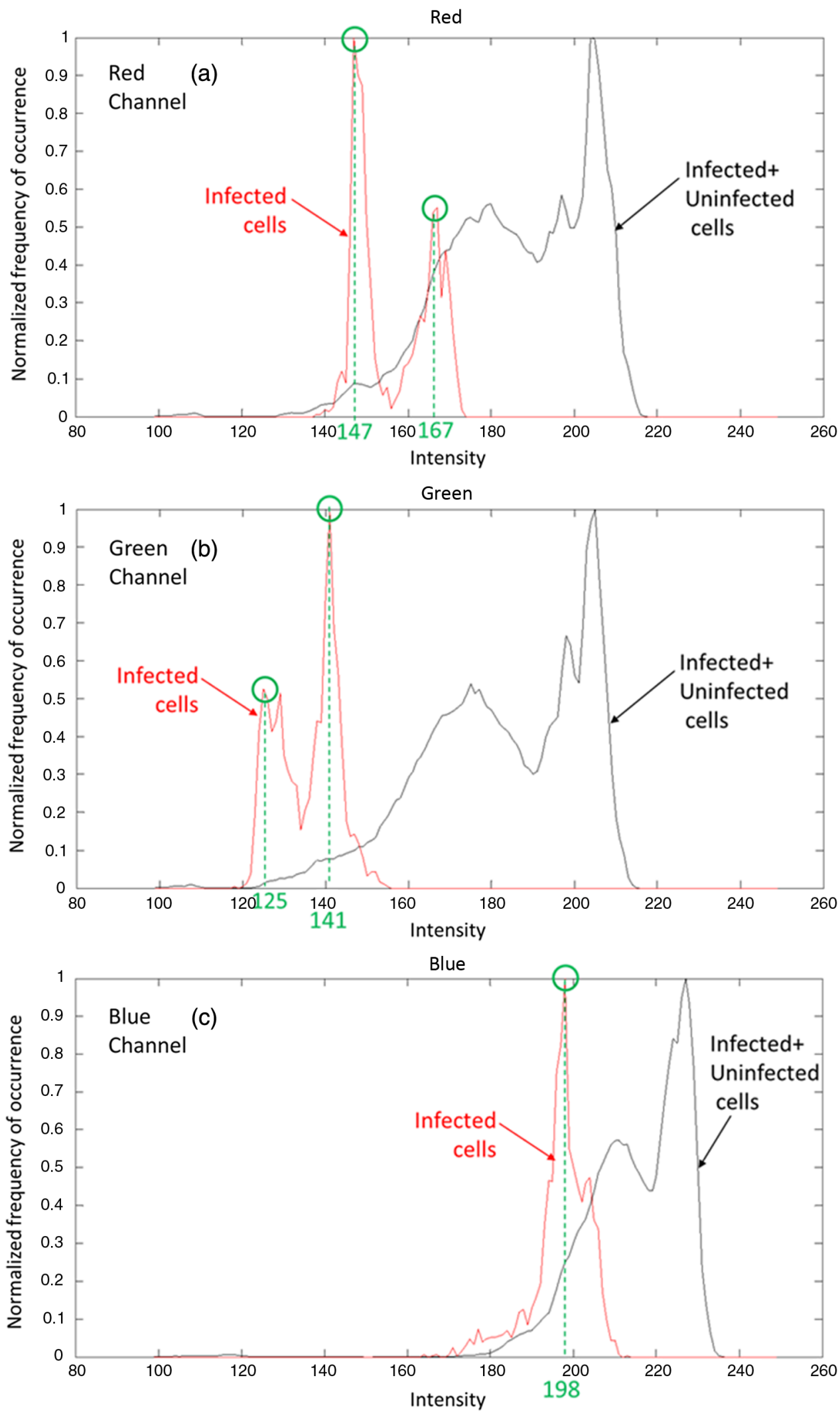

Fig. 19 Histogram of intensity for RGB channels: (a) red, (b) green, and (c) blue channel. The intensity values at the peaks are considered to be the RGB values for identifying the malarial infected cells in the given image acquired by $I_{\text {smart }}$. 


\section{Disclosures}

The authors declare no competing financial interests and conflict of interests.

\section{Acknowledgments}

This work was partially funded by MeitY, Government of India (CEN, IIT Bombay, Project code: 11DIT005). We thank IITBNF, IIT Bombay Central Facility (FDXM, Conductive AFM, Bio-AFM) and TATA Center (IIT Bombay) for providing the required facilities and Molecular Parasitology Lab (IIT Bombay) for the malaria samples. We thank Prof. M. Sarunic (SFU, Burnaby), Prof. M. R. Shenoy (IIT Delhi), N. Tiwary, Dr. N. Punjabi, Farha Memon, Samrat for helpful discussions.

\section{References}

1. D. J. Hartman et al., "Pocket pathologist: a mobile application for rapid diagnostic surgical pathology consultation," J. Pathol. Inf. 5, 10 (2014).

2. C. W. Pirnstill and G. L. Coté, "Malaria diagnosis using a mobile phone polarized microscope," Sci. Rep. 5, 13368 (2015).

3. S. Ekgasit et al., "Elastomeric PDMS planoconvex lenses fabricated by a confined sessile drop technique," ACS Appl. Mater. Interfaces 8(31), 20474-20482 (2016).

4. S. A. Lee and C. Yang, "A smartphone-based chip-scale microscope using ambient illumination," Lab Chip 14, 3056-3063 (2014).

5. M. Madder et al., "E-surveillance in animal health: use and evaluation of mobile tools," Parasitology 139, 1831-1842 (2012).

6. H. Kim et al., "LudusScope: accessible interactive smartphone microscopy for life-science education," PLoS One 11, e0162602 (2016).

7. Y. S. Zhang et al., "A cost-effective fluorescence mini-microscope for biomedical applications," Lab Chip 15, 3661-3669 (2015).

8. Y. L. Sung et al., "Fabricating optical lenses by inkjet printing and heatassisted in situ curing of polydimethylsiloxane for smartphone microscopy," J. Biomed. Opt. 20, 047005 (2015).

9. R. Amarit et al., "High-quality large-magnification polymer lens from needle moving technique and thermal assisted moldless fabrication process," PLoS One 11, e0146414 (2016).

10. C. G. Costa et al., "Optical microsystem design and fabrication for medical image magnification," Microsyst. Technol. 22, 1747-1755 (2016).

11. Z. J. Smith et al., "Cell-phone-based platform for biomedical device development and education applications," PLoS One 6, e17150 (2011).

12. J. S. Cybulski et al., "Foldscope: origami-based paper microscope," PLoS One 9, e98781 (2014).

13. W. M. Lee et al., "Fabricating low cost and high performance elastomer lenses using hanging droplets," Biomed. Opt. Express 5, 1626-1635 (2014).

14. N. A. Switz et al., "Low-cost mobile phone microscopy with a reversed mobile phone camera lens," PLoS One 9, e95330 (2014).

15. Z. D. Popovic et al., "Technique for monolithic fabrication of microlens arrays," Appl. Opt. 27, 1281-1284 (1988).

16. S. Biehl et al., "Refractive microlens fabrication by ink-jet process," J. Sol-Gel Sci. Technol. 13, 177-182 (1998).

17. M. H. Wu et al., "Fabrication of arrays of microlenses with controlled profiles using gray-scale microlens projection photolithography," Langmuir 18, 9312-9318 (2002).

18. P. Zhang et al., "Microlens fabrication using an etched glass master," Microsyst. Technol. 13, 339-342 (2007).

19. B. Karunakaran et al., "Fabrication of nearly hemispherical polymer lenses using water droplets as moulds," IETE Tech. Rev. 33, 54-59 (2015).

20. F. A. Chowdhury and K. J. Chau, "Variable focus microscopy using a suspended water droplet," J. Opt. 14, 055501 (2012).
21. K. Nakakubo et al., "Gallium and polydimethylsiloxane molding for self-organized spherical lens surface fabrication," Appl. Opt. 56, 9900-9906 (2017).

22. M. W. Seo et al., "Simple and low-cost polymer lens fabrication using a pressure-driven micro chamber," in Proc. of 39th Annual Int. Conf. of the IEEE Engineering in Medicine and Biology (EMBC), pp. 37853788 (2017).

23. G. M. Whitesides and S. K. Y. Tang, "Fluidic optics," Proc. SPIE 6329, 63290A (2006).

24. K. Mishra et al., "Optofluidic lens with tunable focal length and asphericity," Sci. Rep. 4, 6378 (2014).

25. B. Karunakaran et al., "Low-cost fabrication of elastomer lenses with real-time control on lens curvature," in Proc. of the 20th Int. Conf. on Miniaturized Systems for Chemistry and Life Sciences ( $\mu$ TAS), Dublin, Ireland, pp. 1057-1058 (2016).

26. A. Tripathi and N. Chronis, "A doublet microlens array for imaging micron-sized objects," J. Micromech. Microeng. 21, 105024 (2011).

27. T. Y. Lee and S. D. Lin, "Dual watermark for image tamper detection and recovery," Pattern Recognit. 41, 3497-3506 (2008).

28. Z. Wang, "Image quality assessment: from error visibility to structural similarity," IEEE Trans. Image Process. 13, 600-612 (2004).

Bhuvaneshwari Karunakaran is a $\mathrm{PhD}$ student in biomedical engineering at Indian Institute of Technology, Bombay, India. She received her $M T$ Tech degree in biomedical engineering from the same institute. Her research currently focuses on development of miniature, low-cost optical imaging platforms for healthcare applications. Her research interests include MEMS/NEMS based sensors, optical imaging, microfluidics, microfabrication, point-of-care diagnostics, and signal processing.

Joseph Tharion received his medical degree from the Government Medical College, Trivandrum, his MTech degree in biomedical engineering, and his PhD in biomedical engineering from Indian Institute of Technology (Bombay). His doctoral work involved developing novel methods for synthesizing metal nanoparticles which can be used in SERS-based biosensors and as theranostic agents. His current research work involves developing device level applications using conducting polymers, in the areas of electrochromics, supercapacitors, and sensors.

Arvind Ramrao Dhawangale is a research associate at Indian Institute of Technology Bombay, India. He received his MTech degree in biomedical engineering from the same institute. He specializes in developing instrumentation for handheld detection systems that include portable surface plasmon resonance and evanescent wavebased optical sensors. His current work focuses on designing highly specific sensors using optical fibers coated with different chemistry, such as gold nanoparticles, disease markers, or polyaniline.

Debjani Paul received her PhD from Indian Institute of Science, Bangalore (India), where she developed a portable thermocycler for DNA amplification. During her postdoctoral research at Curie Institute, Paris, and Cambridge University, she explored several labelfree biosensing techniques. Currently, she is an associate professor at the Department of Biosciences and Bioengineering, Indian Institute of Technology (Bombay). Some of her current work includes paperfluidics, microfluidic cell sorting, and mobile microscopy-based diagnostic platforms.

Soumyo Mukherji received his $\mathrm{PhD}$ in biomedical engineering, University of North Carolina (Chapel Hill, USA). Currently, he is a chair professor in the Department of Biosciences and Bioengineering, Indian Institute of Technology (Bombay). His research focus is in development of inexpensive sensors and sensing platforms for wide-scale deployment in medical and environmental applications. 